\title{
Estado nutricional y composición corporal en escolares de La Serena, Chile
}

\author{
Nutritional status and body composition \\ in school children from La Serena, Chile
}

\begin{abstract}
Objective: To determine nutritional status and body composition of schoolchildren from a school in La Serena city. Material and Methods: a Cross-sectional study in 497 School children between 6 and 15 years. BMI and body composition indicators we calculated. Results: The prevalence of obesity was $19.7 \%$, higher in men $(24.4 \% ; p=0.010)$ and the overweight prevalence was $24.3 \%$, higher in women $(26.7 \% ; p=0.264)$. Excess fat component was $37.6 \%$, more prevalent in the 1 st cycle $(50.0 \% ; p<0.001)$. Deficit of muscular mass was $31.8 \%$, higher in women $(36.2 \%$; $p=0.048)$ and in the 2 nd cycle $(36.9 \% ; p=0.002)$. Regarding the cardiovascular risk, measured by waist circumference, it was $17.7 \%$, being slightly higher in men (18.5\%; $p=0.794)$. Conclusion: There is high prevalence in the variables studied in these school. Following nutritional status and interventions is essential to prevent early onset of non communicable diseases. Key words: Overweight; obesity; body composition; La Serena; school children.
\end{abstract}

María Hortencia Alarcón A. (1) Domingo Antonio Lancellotti G. (1) Andrés Rolando Pedreros L. (2) Claudia Alejandra Bugueño A. (3) Rubén Alejandro Munizaga R.(2)

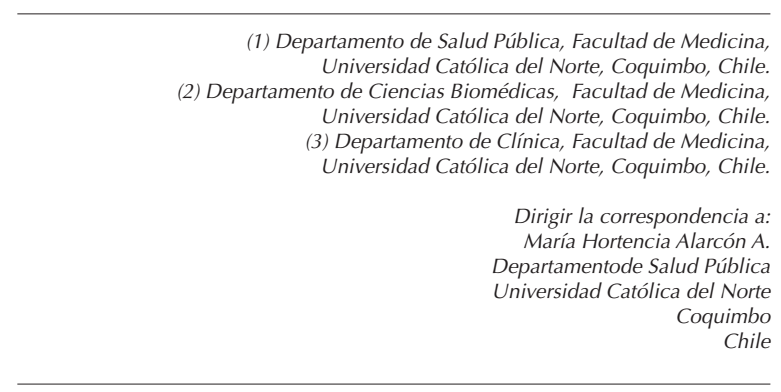

Este trabajo fue recibido el 28 de Abril de 2015, aceptado con modificaciones el 15 de Octubre de 2015 y aceptado para ser publicado el 20 de Marzo de 2016.

\section{INTRODUCCIÓN}

La obesidad es una enfermedad crónica, declarada por la Organización Mundial de la Salud (OMS) como la epidemia mundial del siglo $\mathrm{XXI}$ y considerada uno de los principales problemas de salud pública debido a su tendencia creciente (1). Esta patología se encuentra presente en todos los grupos etarios, cobrando particular preocupación en la población infantil, puesto que los niños que la padecen tienden a mantenerla y aumentarla en la etapa adulta (2) y presentan mayor probabilidad de sufrir a temprana edad resistencia a la insulina, dislipidemia, diabetes tipo 2, hipertensión arterial, problemas osteoarticulares y algunos tipos de cáncer $(3,4)$.

El índice de masa corporal (IMC) es el indicador antropométrico más utilizado para el diagnóstico del estado nutricional en la población escolar (5), sin embargo, no diferencia el componente graso y muscular ni informa sobre la distribución de la grasa corporal (6). Con el perfil actual de obesidad y su asociación con enfermedades no transmisibles, se hace indispensable complementar esta información con otros métodos de medición, que permitan estimar la magnitud y la distribución de la grasa corporal (7). Varios estudios evidencian que el aumento de la grasa corporal es un indicador temprano de riesgo cardiovascular por su correlación directa con la magnitud en la prevalencia de alteraciones metabólicas, y que la medición de perímetros corporales y pliegues cutáneos presentan mayor utilidad en estas asociaciones $(8,9)$.

Con respecto a los perímetros corporales, destaca la importancia de medir la circunferencia de cintura (CC) como indicador de obesidad abdominal, puesto que esta medición se asocia a un perfil lipídico aterogénico y a concentraciones elevadas de insulina, tornándose en una herramienta útil para evaluar el riesgo cardiovascular (RCV) y metabólico $(10,11)$.

Por otro lado, la medición del perímetro braquial (PB) es importante junto con el pliegue tricipital (PT), ya que permiten calcular el área muscular braquial (AMB), como indicador de masa muscular, y el área grasa braquial (AGB), como indicador del compartimento graso, utilizando las fórmulas propuestas por Frisancho $(7,12)$.

En nuestro país la información del estado nutricional de los escolares, proviene de la Junta Nacional Auxilio Escolar y Beca (JUNAEB), datos que se obtienen de las escuelas, y que corresponden a los escolares de $1^{\circ}$ básico (edades entre 6 y 7 años) y $1^{\circ}$ medio (edades entre 14 y 15 años), y que indican al año 2013 una alta prevalencia de obesidad en ambos grupos, 
llegando a un 25,3\% y 12,3\%, respectivamente (13), situación similar a lo encontrado en diferentes estudios realizados en nuestro país para esta población (14-18).

En la ciudad de La Serena de la Región de Coquimbo faltan estudios sobre la situación nutricional en estos grupos poblacionales, por esta razón este estudio tuvo por objetivo determinar el estado nutricional y la composición corporal de los escolares de un colegio de esta ciudad, según sexo y ciclo de enseñanza básica.

\section{SUJETOS Y MÉTODOS}

Se realizó un estudio de corte transversal en el segundo semestre de 2011 en escolares del colegio particular subvencionado Adventista "Maranata", ubicado en la ciudad de La Serena, IV Región de Coquimbo - Chile. Este estudio fue solicitado por el colegio y el Centro de Salud Familiar Cardenal Caro, y que llevó a conformar una red de trabajo intersectorial con la Universidad Católica del Norte para obtener los datos, los que se usaron como línea base para aplicar la estrategia de promoción de la salud en este establecimiento educacional.

Participaron un total de 497 escolares en edades de 6 a 15 años, correspondientes al $1^{\circ}$ ciclo ( $1^{\circ}$ año básico a $4^{\circ}$ año básico: edades de 6 a 9 años) y $2^{\circ}$ ciclo ( $5^{\circ}$ año básico a $8^{\circ}$ año básico: edades de 10 a 15 años) de enseñanza básica, representando al 96,9\% del universo.

Estos escolares procedían de diferentes sectores de la ciudad de La Serena y de la ciudad de Coquimbo. En lo socioeconómico pertenecían al grupo medio bajo, según el índice que utiliza la JUNAEB para determinar vulnerabilidad escolar (IVE: 54,33) (19). Se excluyeron aquellos escolares con causas secundarias de malnutrición por exceso (Síndrome de Prader Willi, entre otros) de enfermedades crónicas o malformaciones congénitas mayores.

Las variables estudiadas fueron el estado nutricional y la composición corporal, mediante los siguientes indicadores antropométricos; talla, peso, perímetro braquial (PB), pliegue tricipital (PT) y circunferencia de cintura (CC). Con estas mediciones se obtuvo la Talla/edad (T/E), el Área Grasa Braquial (AGB), Área Muscular Braquial (AMB), Circunferencia Cintura/ Edad (CC/E), IMC/E. Cada indicador y variable fueron descritas según sexo y ciclo de enseñanza básica.

Para realizar las mediciones se utilizaron instrumentos cali- brados; para la talla y peso se utilizó una balanza mecánica con tallímetro adosado marca SECA, la balanza con una precisión de $100 \mathrm{~g}$. y el tallímetro de 0,1 cm; para la circunferencia de cintura y perímetro braquial se utilizó una cinta métrica inextensible marca SECA con una precisión de $1 \mathrm{~mm}$; para determinar el pliegue tricipital se usó un Caliper Lange con precisión de 1 $\mathrm{mm}$. A partir del perímetro braquial y el pliegue tricipital, se obtuvo el Perímetro Muscular Braquial (PMB, en mm), AMB (en $\mathrm{mm} 2$ ) y el $A G B$ (en $\mathrm{mm} 2$ ), utilizando las fórmulas propuestas por Frisancho (12).

El estado nutricional se clasificó utilizando los parámetros antropométricos según la norma técnica del Ministerio de Salud (MINSAL) (5); índice de Masa Corporal según Edad (IMC/Edad), donde la población de referencia para IMC y talla es la propuesta por el Center for Disease Control de EEUU (CDC/NCHS).

La medición de la CC se realizó por encima de las crestas ilíacas, y se consideró "aumentada" cuando fue > p 90 para la edad y sexo, de acuerdo al criterio de diagnóstico de Cook (20). En este estudio la CC clasificada como aumentada se consideró como riesgo cardiovascular (RCV). Todas las mediciones se realizaron utilizando técnicas internacionalmente aceptadas y con personal capacitado y entrenado (académicos de la carrera de Nutrición y Dietética de la Universidad Católica del Norte). El protocolo fue aprobado por el Comité de Bioética de la Facultad de Medicina de la Universidad Católica del Norte y se obtuvo el consentimiento informado de los padres y de los escolares previo a efectuar las mediciones.

Se utilizó una estadística descriptiva (promedio + desviación estándar; distribución de frecuencias absolutas y porcentajes), la comparación de medias para grupos independientes se realizó mediante la prueba de $\mathrm{t}$ de student mientras que las frecuencias se compararon con la prueba de Chi-cuadrado. El nivel de significancia estadística empleado fue 5\%. Para el análisis estadístico se utilizó el programa XLSTAT®.

\section{RESULTADOS}

De los 497 escolares considerados en el estudio el 51,1\% fueron varones. La edad promedio fue 10,6 + 2,4 años, siendo de $8,0+1,2$ años en el $1^{\circ}$ ciclo y de $12,2 \pm 1,2$ años en el $2^{\circ}$ ciclo. Según el sexo no hubo diferencias significativas para la mayoría de las variables cuantificadas, a excepción del pliegue tricipital que resultó ser mayor en mujeres tanto en el $1^{\circ}$ ciclo

\section{TABLA 1}

Características de los escolares según sexo y ciclo de enseñanza básica.

\begin{tabular}{|c|c|c|c|c|c|c|c|c|}
\hline \multirow[t]{3}{*}{ Características } & & $1^{\circ} \mathrm{Ciclo} \mathrm{Básico}$ & & \multicolumn{5}{|c|}{$2^{\circ}$ Ciclo Básico } \\
\hline & $\begin{array}{l}\text { Varones } \\
(\mathrm{N}=98)\end{array}$ & $\begin{array}{l}\text { Mujeres } \\
(\mathrm{N}=90)\end{array}$ & $\begin{array}{c}\text { Total } \\
(\mathrm{N}=188)\end{array}$ & valor $\mathrm{p}^{*}$ & $\begin{array}{l}\text { Varones } \\
(\mathrm{N}=156)\end{array}$ & $\begin{array}{l}\text { Mujeres } \\
(\mathrm{N}=153)\end{array}$ & $\begin{array}{c}\text { Total } \\
(\mathrm{N}=309)\end{array}$ & valor $\mathrm{p}^{*}$ \\
\hline & $x \pm D E$ & $x \pm D E$ & $x \pm D E$ & & $x \pm D E$ & $x \pm D E$ & $x \pm D E$ & \\
\hline Edad (años) & $8,0 \pm 1,2$ & $8,0 \pm 1,3$ & $8,0 \pm 1,2$ & 0,733 & $12,2 \pm 1,2$ & $12,2 \pm 1,2$ & $12,2 \pm 1,2$ & 0,698 \\
\hline Peso (kg) & $29,9 \pm 6,8$ & $30,6 \pm 8,6$ & $30,2 \pm 7,7$ & 0,543 & $48,5 \pm 12,8$ & $48,5 \pm 12,7$ & $48,5 \pm 12,8$ & 0,971 \\
\hline Talla (m) & $1,26 \pm 0,08$ & $1,27 \pm 0,09$ & $1,27 \pm 0,09$ & 0,468 & $1,51 \pm 0,11$ & $1,50 \pm 0,08$ & $1,51 \pm 0,09$ & 0,638 \\
\hline IMC $\left(\mathrm{kg} / \mathrm{m}^{2}\right)$ & $18,6 \pm 2,8$ & $18,5 \pm 3,0$ & $18,5 \pm 2,9$ & 0,969 & $21,0 \pm 3,7$ & $21,2 \pm 4,2$ & $21,1 \pm 3,9$ & 0,665 \\
\hline $\mathrm{CC}(\mathrm{cm})$ & $62,6 \pm 7,9$ & $63,1 \pm 7,7$ & $62,8 \pm 7,8$ & 0,671 & $73,5 \pm 9,6$ & $73,2 \pm 11,2$ & $73,3 \pm 10,4$ & 0,842 \\
\hline PB (cm) & $20,6 \pm 2,8$ & $20,9 \pm 2,8$ & $20,7 \pm 2,8$ & 0,457 & $24,2 \pm 3,7$ & $24,3 \pm 3,5$ & $24,3 \pm 3,6$ & 0,719 \\
\hline PT (mm) & $16,6 \pm 5,7$ & $18,9 \pm 5,6$ & $17,7 \pm 5,7$ & 0,007 & $19,3 \pm 7,3$ & $22,8 \pm 7,3$ & $21,0 \pm 7,5$ & $<0,001$ \\
\hline
\end{tabular}

*Prueba t de Student

IMC =Índice de Masa Corporal; $C C=$ Circunferencia de Cintura; PB= Perímetro Braquial; $\mathrm{PT}=$ Pliegue Tricipital. 


\section{FIGURA 1}

Clasificación de la talla de los escolares.

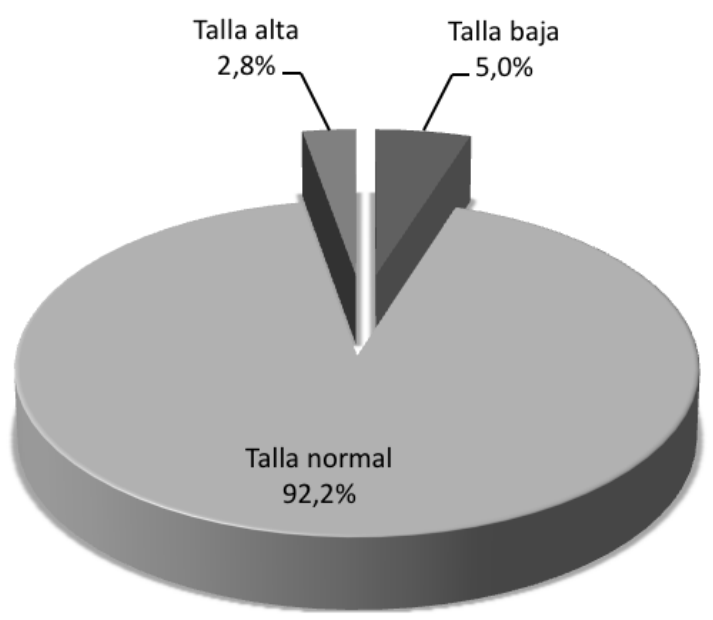

FIGURA 2

Clasificación de la talla baja y talla alta de los escolares según sexo y ciclo de enseñanza básica.

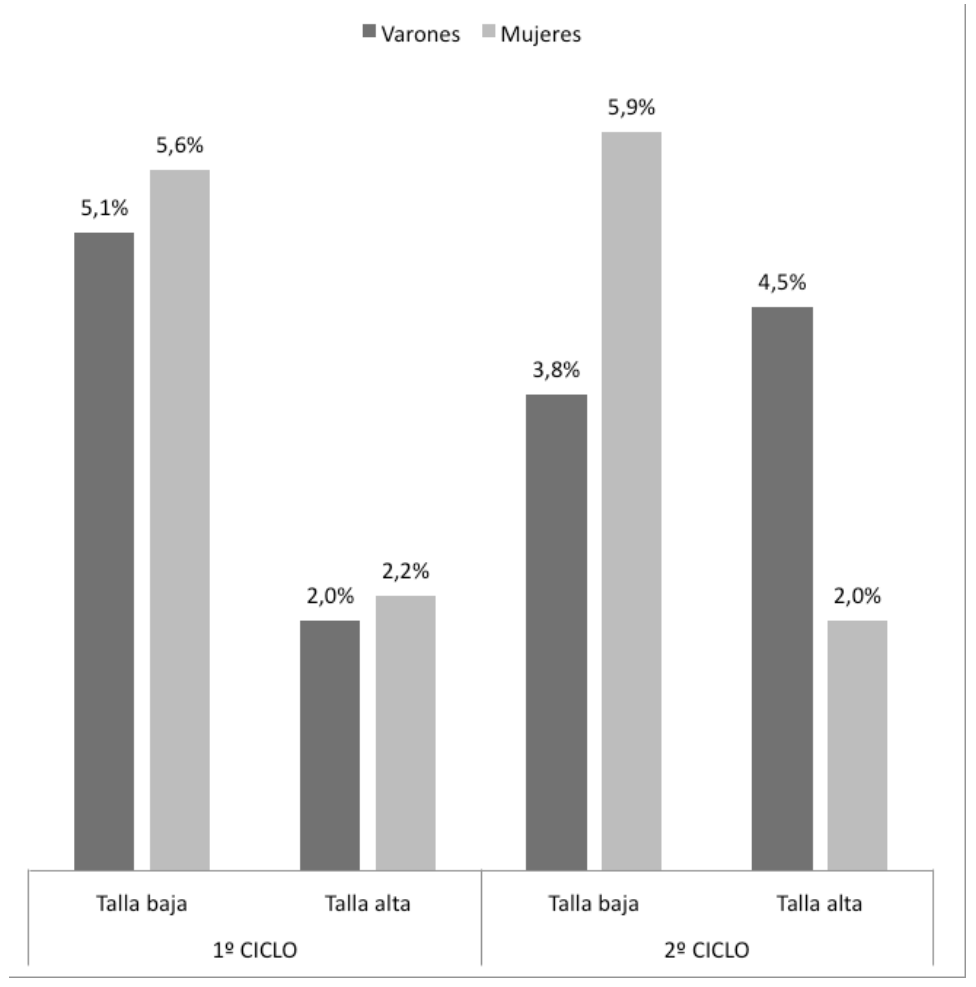


$(p=0,007)$ como en el $2^{\circ}$ ciclo $(p<0,001)$ (tabla 1$)$.

El $92,2 \%$ de los escolares presentó una talla normal (figura 1), no observando diferencias significativas entre los ciclos. En ambos ciclos las mujeres presentaron siempre mayor porcentaje de talla baja (figura 2), situación que aumentó con la edad en contraste a los varones quienes aumentaron en talla alta, tendencias que no fueron significativas $(p=0,985$ y $\mathrm{p}=0,537$, respectivamente).

\section{TABLA 2}

Clasificación del estado nutricional de los escolares según sexo y ciclo de enseñanza básica.

\begin{tabular}{|c|c|c|c|c|c|c|c|c|c|}
\hline Clasificación & & $1^{\circ}$ ciclo & & & $2^{\circ}$ ciclo & & Total & Total & Total \\
\hline $\begin{array}{c}\text { Estado } \\
\text { Nutricional }\end{array}$ & $\begin{array}{c}\text { Varones } \\
(\mathrm{n}=98) \\
(\%)\end{array}$ & $\begin{array}{c}\text { Mujeres } \\
(\mathrm{n}=90) \\
(\%)\end{array}$ & $\begin{array}{c}\text { Total } 1^{\circ} \text { ciclo } \\
(\mathrm{n}=188) \\
(\%)\end{array}$ & $\begin{array}{c}\text { Varones } \\
(\mathrm{n}=156) \\
(\%)\end{array}$ & $\begin{array}{c}\text { Mujeres } \\
(\mathrm{n}=153) \\
(\%)\end{array}$ & $\begin{array}{c}\text { Total } 2^{\circ} \text { ciclo } \\
(\mathrm{n}=309) \\
(\%)\end{array}$ & $\begin{array}{c}\text { Varones } \\
(\mathrm{n}=254) \\
(\%)\end{array}$ & $\begin{array}{c}\text { Mujeres } \\
(\mathrm{n}=243) \\
(\%)\end{array}$ & $(n=497)$ \\
\hline BP & $\begin{array}{c}1 \\
(1,0)\end{array}$ & $\begin{array}{c}1 \\
(1,1)\end{array}$ & $\begin{array}{c}2 \\
(1,1)\end{array}$ & $\begin{array}{c}4 \\
(2,6)\end{array}$ & $\begin{array}{c}3 \\
(2,0)\end{array}$ & $\begin{array}{c}7 \\
(2,3)\end{array}$ & $\begin{array}{c}5 \\
(2,0)\end{array}$ & $\begin{array}{c}4 \\
(1,6)\end{array}$ & $\begin{array}{c}9 \\
(1,8)\end{array}$ \\
\hline $\mathrm{N}$ & $\begin{array}{c}47 \\
(48,0)\end{array}$ & $\begin{array}{c}46 \\
(51,1)\end{array}$ & $\begin{array}{c}93 \\
(49,5)\end{array}$ & $\begin{array}{c}84 \\
(53,8)\end{array}$ & $\begin{array}{c}92 \\
(60,1)\end{array}$ & $\begin{array}{c}176 \\
(57,0)\end{array}$ & $\begin{array}{c}131 \\
(51,6)\end{array}$ & $\begin{array}{c}138 \\
(56,8)\end{array}$ & $\begin{array}{c}269 \\
(54,1)\end{array}$ \\
\hline$S$ & $\begin{array}{c}18 \\
(18,4)\end{array}$ & $\begin{array}{c}27 \\
(30,0)\end{array}$ & $\begin{array}{c}45 \\
(23,9)\end{array}$ & $\begin{array}{c}38 \\
(24,4)\end{array}$ & $\begin{array}{c}38 \\
(24,8)\end{array}$ & $\begin{array}{c}76 \\
(24,6)\end{array}$ & $\begin{array}{c}56 \\
(22,0)\end{array}$ & $\begin{array}{c}65 \\
(26,7)\end{array}$ & $\begin{array}{c}121 \\
(24,3)\end{array}$ \\
\hline $\mathrm{O}$ & $\begin{array}{c}32 \\
(32,7)^{*}\end{array}$ & $\begin{array}{c}16 \\
(17,8)\end{array}$ & $\begin{array}{c}48 \\
(25,5)^{*}\end{array}$ & $\begin{array}{c}30 \\
(19,2)\end{array}$ & $\begin{array}{c}20 \\
(13,1)\end{array}$ & $\begin{array}{c}50 \\
(16,2)\end{array}$ & $\begin{array}{c}62 \\
(24,4)^{*}\end{array}$ & $\begin{array}{c}36 \\
(14,8)\end{array}$ & $\begin{array}{c}98 \\
(19,7)\end{array}$ \\
\hline
\end{tabular}

${ }^{*} \mathrm{p}<0.05$ (Test Chi cuadrado)

$\mathrm{BP}=$ bajo peso; $\mathrm{N}=$ Normal; $\mathrm{S}=$ sobrepeso; $\mathrm{O}=$ obeso.

\section{FIGURA 3}

Estado nutricional de los escolares según ciclo de enseñanza básica.

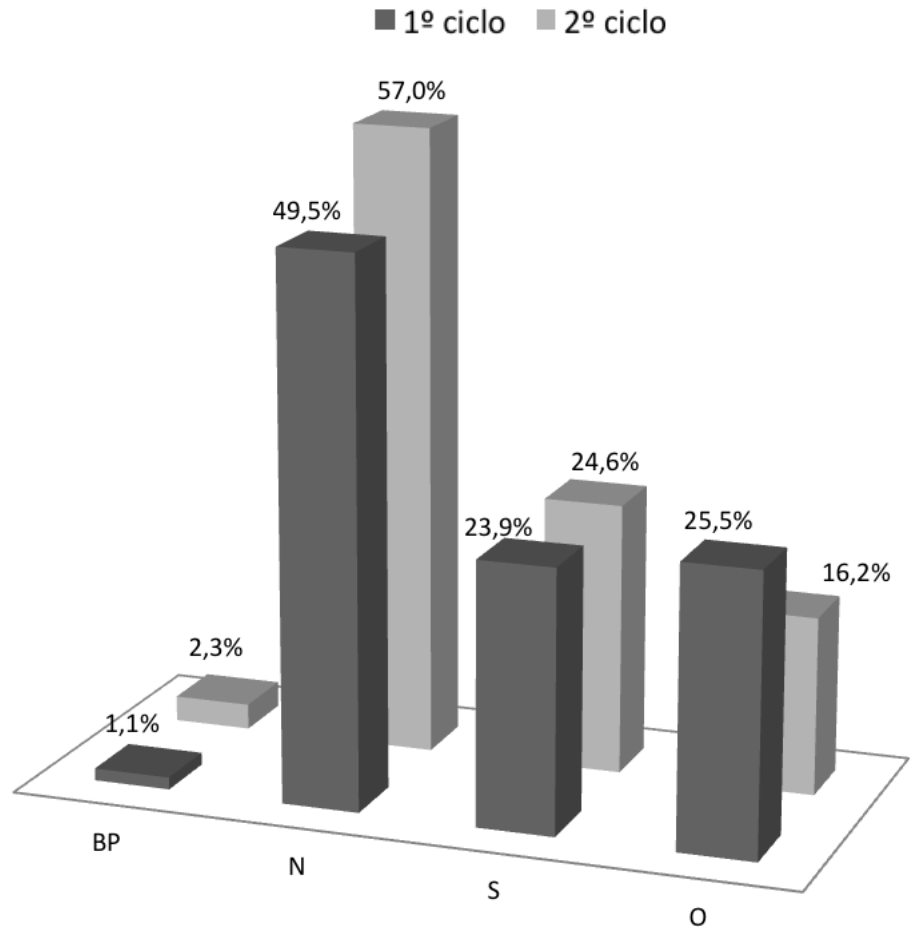

$\mathrm{BP}=$ bajo peso; $\mathrm{N}=$ normal $; \mathrm{S}=$ sobrepeso; $\mathrm{O}=$ obeso. 
La prevalencia total de malnutrición por exceso fue $44,0 \%$, alcanzando la obesidad $19,7 \%$ y el sobrepeso $24,3 \%$. La obesidad fue mayor en varones $(p=0,010)$ mientras que el sobrepeso lo fue en las mujeres, esto último no significativo $(p=0,264)$ (tabla 2$)$. Por ciclos de enseñanza básica, la malnutrición por exceso fue 8,6 puntos porcentuales mayor en los escolares de menor edad, pero no significativo $(p=0,072)$, con la obesidad mayor en el $1^{\circ}$ ciclo $(p=0,015)$ y el sobrepeso prácticamente sin variación $(p=0,953)$ (figura 3 ). La obesidad siempre fue mayor en los hombres pero sólo significativa en el primer ciclo ( $p=0,030$ vs. $p=0,188)$, mientras que el sobrepeso fue mayor en las mujeres del $1^{\circ}$ ciclo pero no significativo $(p=0,090)$, y en el $2^{\circ}$ ciclo no hubo diferencias entre sexos $(p=0,972)$.

El bajo peso alcanzó el 1,8\% de los escolares, porcentaje que tiende aumentar en el segundo ciclo pero no significativamente $(p=0,530)$, bajo peso que, en general presentó mínimas diferencias por sexo $\left(p=0,515\right.$ y $p=0,979$, para el $1^{\circ}$ y $2^{\circ}$ ciclo, respectivamente) (tabla 2 ).

El 17,7\% de los escolares presentó RCV, el que en general fue más prevalente en los varones y en el $1^{\circ}$ ciclo pero no significativo ( $p=0,794$ y $p=0,736$, respectivamente)(tabla3).

El indicador de masa muscular (AMB) mostró una prevalencia de déficit en $31,8 \%$ de la población general de escolares, y que según el sexo fue significativamente mayor en las mujeres $(p=0,048)$ y según grupo de edad en el $2^{\circ}$ ciclo $(p=0,002)$, valores que fueron significativos en hombres $(p=0,014)$ pero no en mujeres $(p=0,092)$ (tabla 3$)$.

El AGB indicó un exceso de grasa en $37,6 \%$ de los escolares, más prevalente en los varones, pero no significativo $(p=0,098)$, y según el ciclo fue significativamente más prevalente en el $1^{\circ}$ ciclo $(p<0,001)$ y en ambos sexos $(p=0,009$ y $p=0,001$ para hombres y mujeres, respectivamente) (tabla 3).

\section{DISCUSIÓN}

En nuestro país, este tipo de información nutricional es escasa y heterogénea dado los distintos grupos etarios estudiados y con diferentes referencias para el levantamiento y procesamiento de la información, todos aspectos relevantes al momento de establecer comparaciones. En el caso de los resultados de este estudio, sin bien no pueden ser extrapolados al resto de la población escolar, por contemplar escolares de sólo un colegio, son relevantes ya que muestran una realidad local, considerando al 96,9\% de los escolares de $1^{\circ}$ a $8^{\circ}$ básico, en edades de 6 a 15 años. Por otra parte, los resultados encontrados en estos escolares se relacionan con el aumento de la obesidad, enfermedades cardiovasculares y diabetes tipo 2 reportada en la población mayor de 15 años de nuestro país (21), lo que sustenta la necesidad de realizar otros estudios considerando un número de establecimientos educacionales representativos de la Región, y que incorporen las diferentes edades de los escolares.

Los resultados en este estudio confirman una alta prevalencia de obesidad $(19,7 \%)$ y sobrepeso $(24,3 \%)$ en esta

TABLA 3

Distribución del RCV, AMB y AGB de los escolares según sexo y ciclo de enseñanza básica.

\begin{tabular}{|c|c|c|c|c|c|c|c|c|c|}
\hline \multirow[b]{2}{*}{ Indicadores } & \multicolumn{3}{|c|}{$1^{\circ}$ Ciclo } & \multicolumn{3}{|c|}{$2^{\circ}$ Ciclo } & \multirow[b]{2}{*}{$\begin{array}{c}\text { Total } \\
\text { Varones } \\
(\mathbf{n}=\mathbf{2 5 4}) \\
(\%)\end{array}$} & \multirow[b]{2}{*}{$\begin{array}{c}\text { Total } \\
\text { Mujeres } \\
(\mathbf{n}=\mathbf{2 4 3}) \\
(\%)\end{array}$} & \multirow[b]{2}{*}{$\begin{array}{c}\text { TOTAL } \\
\text { Ambos } \\
\text { Ciclos } \\
(\mathrm{n}=497) \\
(\%)\end{array}$} \\
\hline & $\begin{array}{c}\text { Varones } \\
(\mathbf{n}=98) \\
(\%)\end{array}$ & $\begin{array}{c}\text { Mujeres } \\
(\mathbf{n}=90) \\
(\%)\end{array}$ & $\begin{array}{c}\text { Total } \\
1^{\circ} \text { Ciclo } \\
(n=188) \\
(\%)\end{array}$ & $\begin{array}{c}\text { Varones } \\
(\mathrm{n}=156) \\
(\%)\end{array}$ & $\begin{array}{c}\text { Mujeres } \\
(\mathrm{n}=153) \\
(\%)\end{array}$ & $\begin{array}{c}\begin{array}{c}\text { Total } \\
2^{\circ} \text { Ciclo } \\
(n=309) \\
(\%)\end{array} \\
(\%)\end{array}$ & & & \\
\hline \multicolumn{10}{|c|}{ RCV } \\
\hline Sin RCV & $\begin{array}{c}76 \\
(77,6)\end{array}$ & $\begin{array}{c}77 \\
(85,6)\end{array}$ & $\begin{array}{c}153 \\
(81,4)\end{array}$ & $\begin{array}{c}131 \\
(84,0)\end{array}$ & $\begin{array}{c}125 \\
(81,7)\end{array}$ & $\begin{array}{c}256 \\
(82,8)\end{array}$ & $\begin{array}{c}207 \\
(81,5)\end{array}$ & $\begin{array}{c}202 \\
(83,1)\end{array}$ & $\begin{array}{c}409 \\
(82,3)\end{array}$ \\
\hline $\mathbf{C o n}_{\mathbf{R C V}}{ }^{\dagger}$ & $\begin{array}{c}22 \\
(22,4)\end{array}$ & $\begin{array}{c}13 \\
(14,4)\end{array}$ & $\begin{array}{c}35 \\
(18,6)\end{array}$ & $\begin{array}{c}25 \\
(16,0)\end{array}$ & $\begin{array}{c}28 \\
(18,3)\end{array}$ & $\begin{array}{c}53 \\
(17,2)\end{array}$ & $\begin{array}{c}47 \\
(18,5)\end{array}$ & $\begin{array}{c}41 \\
(16,9)\end{array}$ & $\begin{array}{c}88 \\
(17,7)\end{array}$ \\
\hline \multicolumn{10}{|c|}{ AMB } \\
\hline Déficit AMB & $\begin{array}{c}18 \\
(18,4)\end{array}$ & $\begin{array}{c}26 \\
(28,9)\end{array}$ & $\begin{array}{c}44 \\
(23,4)\end{array}$ & $\begin{array}{c}52 \\
(33,3)^{*}\end{array}$ & $\begin{array}{c}62 \\
(40,5)\end{array}$ & $\begin{array}{c}114 \\
(36,9)^{*}\end{array}$ & $\begin{array}{c}70 \\
(27,6)\end{array}$ & $\begin{array}{c}88 \\
(36,2)^{*}\end{array}$ & $\begin{array}{c}158 \\
(31,8)\end{array}$ \\
\hline Normal AMB & $\begin{array}{c}75 \\
(76,5)\end{array}$ & $\begin{array}{c}63 \\
(70,0)\end{array}$ & $\begin{array}{c}138 \\
(73,4)\end{array}$ & $\begin{array}{c}95 \\
(60,9)\end{array}$ & $\begin{array}{c}87 \\
(56,9)\end{array}$ & $\begin{array}{c}182 \\
(58,9)\end{array}$ & $\begin{array}{c}170 \\
(66,9)\end{array}$ & $\begin{array}{c}150 \\
(61,7)\end{array}$ & $\begin{array}{c}320 \\
(64,4)\end{array}$ \\
\hline Exceso AMB & $\begin{array}{c}5 \\
(5,1) \\
\end{array}$ & $\begin{array}{c}1 \\
(1,1)\end{array}$ & $\begin{array}{c}6 \\
(3,2)\end{array}$ & $\begin{array}{c}9 \\
(5,8)\end{array}$ & $\begin{array}{c}4 \\
(2,6)\end{array}$ & $\begin{array}{c}13 \\
(4,2)\end{array}$ & $\begin{array}{c}14 \\
(5,5)\end{array}$ & $\begin{array}{c}5 \\
(2,1)\end{array}$ & $\begin{array}{c}19 \\
(3,8)\end{array}$ \\
\hline \multicolumn{10}{|c|}{ AGB } \\
\hline Normal AGB & $\begin{array}{c}47 \\
(48,0)\end{array}$ & $\begin{array}{c}47 \\
(52,2)\end{array}$ & $\begin{array}{c}94 \\
(50,0)\end{array}$ & $\begin{array}{c}102 \\
(65,4)\end{array}$ & $\begin{array}{c}114 \\
(74,5)\end{array}$ & $\begin{array}{c}216 \\
(69,9)\end{array}$ & $\begin{array}{c}149 \\
(58,7)\end{array}$ & $\begin{array}{c}161 \\
(66,3)\end{array}$ & $\begin{array}{c}310 \\
(62,4)\end{array}$ \\
\hline Exceso AGB & $\begin{array}{c}51 \\
(52,0)^{*}\end{array}$ & $\begin{array}{c}43 \\
(47,8)^{* *}\end{array}$ & $\begin{array}{c}94 \\
(50,0)^{* *}\end{array}$ & $\begin{array}{c}54 \\
(34,6)\end{array}$ & $\begin{array}{c}39 \\
(25,5)\end{array}$ & $\begin{array}{c}93 \\
(30,1)\end{array}$ & $\begin{array}{c}105 \\
(41,3)\end{array}$ & $\begin{array}{c}82 \\
(33,7)\end{array}$ & $\begin{array}{c}187 \\
(37,6)\end{array}$ \\
\hline
\end{tabular}

\footnotetext{
${ }^{*} \mathrm{p}<0.05$ (Test Chi cuadrado)

** $\mathrm{p}<0.001$ (Test Chi cuadrado)

$\mathrm{RCV}=$ riesgo cardiovascular; $\mathrm{AMB}=$ área muscular braquial; $\mathrm{AGB}=$ área grasa braquial

$+C o n R C V=$ Clasificado con circunferencia de cintura $>$ percentil 90 según edad y sexo.
} 
población escolar, lo que evidencia una vez más la situación que se observa de esta prevalencia de malnutrición por exceso en esta población en nuestro país $(14,15)$.

En cuanto a la prevalencia de obesidad encontrada en la población del $1^{\circ}$ ciclo de enseñanza básica ( $1^{\circ}$ año básico a $4^{\circ}$ año básico) alcanzó $25,5 \%$ siendo similar a lo reportado a nivel nacional en el grupo de escolares de primer año básico, según los datos entregados por JUNAEB (25,3\%), sin embargo, la prevalencia de obesidad del $2^{\circ}$ ciclo de enseñanza básica ( $5^{\circ}$ año básico a $8^{\circ}$ año básico) comparada con el reporte nacional en escolares de primero medio, fue mayor $(16,2 \%$ vs $12,3 \%$, respectivamente) (13). Esta diferencia podría estar dada por el uso de distintas referencias (22), no obstante, y dado que a nivel nacional sólo se consideraron escolares de primero medio y en este estudio escolares de quinto a octavo básico, diferencia que también podría estar relacionado con el desarrollo puberal. Sin embargo ambos estudios concuerdan en que la prevalencia de obesidad es mayor a menor edad y en varones, situación que se corrobora en otro estudio realizado en escolares en Chile (18).

La prevalencia de sobrepeso fue discretamente mayor en el $2^{\circ}$ ciclo $(23,9 \% \mathrm{v} / \mathrm{s} 24,6 \%)$, lo que difiere con lo reportado por Ulloa y cols., que indicaron una disminución a mayor edad para luego aumentar desde los 14 años, aunque concuerda con este estudio en que la prevalencia de sobrepeso era mayor en mujeres (22).

La situación epidemiológica actual nos obliga a poner el énfasis en obtener información sobre el exceso de peso, no obstante en estas poblaciones cobra importancia el control periódico de bajo peso y talla baja.

A nivel nacional la prevalencia de bajo peso es menor en los escolares de mayor edad $2,0 \%$ en niños de primero básico a $0,7 \%$ en los de primero medio, situación similar en ambos sexos ( $M: 1,8 \%$ a $0,5 \%$ y $V: 2,2 \%$ a $0,9 \%$ ) (13), lo que difiere con lo encontrado en este estudio donde la prevalencia aumentó con la edad ( $1^{\circ}$ ciclo: $1,1 \%$ a $2^{\circ}$ ciclo: $2,3 \%$ ) y en ambos sexos ( $M$ : $1,1 \%$ a $2,0 \%$ y $V: 1,0 \%$ a $2,6 \%)$, diferencias que una vez más pueden estar dadas por el uso de distintas referencias para la clasificación del estado nutricional, por lo que parece necesario estandarizar el uso de una referencia (23). La literatura también nos indica que estas diferencias se pueden presentar al comparar el diagnóstico nutricional basado con datos recolectados por personal no entrenado con aquellos obtenidos en condiciones estandarizadas previamente $(24,25)$. Un reporte realizado por Eyzaguirre y cols. en niños de 6 a 18 años de nivel socioeconómico alto, encontraron una mayor prevalencia de bajo peso $(5,6 \%)$, posiblemente por la diferente situación socioeconómica de las poblaciones estudiadas, sin embargo concuerda con este estudio en que esta prevalencia aumenta a mayor edad. Además, reportaron $91,7 \%$ de niños con talla normal, 3,0\% con talla baja y $5,3 \%$ con talla alta (16), datos que si bien concuerdan más con lo encontrado en este estudio en lo que se refiere a la prevalencia de talla normal $(92,2 \%)$, se contraponen y se invierten en la talla baja respecto de la talla alta $(5,0 \%$ y $2,8 \%$, respectivamente), diferencias que igualmente pueden estar dadas por diferencias en el nivel socioeconómico, puesto que la talla es un buen indicador de la calidad del ambiente en el cual ha vivido el niño. Al comparar esta prevalencia de talla baja de la población general con los resultados nacionales concuerdan en que a mayor edad la talla baja aumenta en las mujeres y disminuye en los varones (13), hecho que podría tener su explicación en la detención fisiológica del crecimiento ligado a la menarquía y desarrollo puberal precoz (26). De acuerdo a lo reportado a nivel nacional la prevalencia de talla baja fue mayor en este estudio en todos los casos, diferencias que también pueden estar dadas por el uso de distintas referencias al momento de evaluar este indicador. Cabe mencionar que en la metodología utilizada en nuestro estudio no se clasificó a los escolares y adolescentes según el estadio de desarrollo de Tanner, por ser un estudio con una mirada de salud pública y no de caso individual, de todos modos podría limitar las conclusiones que se puedan obtener en el grupo de mayor edad.

De acuerdo a lo reportado por Kain y cols en escuelas de Santiago de Chile en niños de 4 a 7 años, la prevalencia RCV (CC > p 90) fue de 10,2\%, y más prevalente en mujeres (27), ambos datos difirieren con lo encontrado en este estudio en escolares del $1^{\circ}$ ciclo (edad entre 6 y 9 años), que indica que esta población presenta a muy temprana edad una alta prevalencia de RCV $(18,6 \%)$ y mayor en varones.

Mardones y cols encontraron 20,9\% de RCV en escolares chilenos de $5^{\circ}$ y $6^{\circ}$ año básico (promedio de edad: 11,4 + 1 años) prevalencia mayor a la encontrada en este estudio (17, $2 \%$ ) en los escolares del $2^{\circ}$ ciclo (promedio de edad 12,2 \pm 1,2 años), lo que podría explicarse porque en este estudio se consideró escolares de $5^{\circ}$ a $8^{\circ}$ año básico, no obstante concordaron en que esta prevalencia es mayor en mujeres (15). En otro estudio realizado en los Estados Unidos, Cook y cols., reportaron para adolescentes de 12 a 19 años 9,8\% de CC > p90, cifra mucho menor a lo encontrado en el presente estudio en escolares de edades similares, diferencias que pueden estar asociadas a las prevalencias de malnutrición por exceso que en este estudio alcanzó $40,8 \%$ vs. 29,0\% entregada por Cook y cols. (20). Se debe considerar que el trabajo de Cook y cols posee una diferencia de más de 15 años con el presente estudio, lo que evidencia la alarmante situación epidemiológica nutricional en que estarían actualmente los niños de este estudio.

Los resultados del compartimento graso y masa muscular, revelan un dismorfismo sexual en la población en estudio, el cual se hace visible por valores significativamente más altos del pliegue tricipital en mujeres respecto a los varones, tanto en el $1^{\circ}$ y $2^{\circ}$ ciclo, $(P=0,007, P<0,001$ respectivamente), y de igual forma en los valores de $A M B$ que fueron menores en mujeres, resultados que coinciden con estudios realizados en población adolescente $(28,29)$. Dada la importancia de estos indicadores como parámetros de estimación de los componentes graso y magro (30), se confirma que las mujeres presentaron un mayor componente graso (PT) y menor componente muscular, el cual se podría relacionar con el sobrepeso, y en el caso de los varones la mayor prevalencia de obesidad se relacionaría con la mayor prevalencia de AGB detectada.

Estas discrepancias en la composición corporal, son similares a los resultados encontrados en la literatura, las que se atribuyen principalmente a las diferencias hormonales en la pubertad (31). Esto último refleja la importancia de incorporar mediciones de perímetro y pliegues cutáneos en la determinación de la composición corporal, la cual resulta imprescindible para comprender el efecto de la alimentación, el crecimiento, el ejercicio físico (32), las enfermedades y otros factores, en el organismo (33).

El importante déficit de masa muscular encontrado en estos escolares $(31,8 \%)$, se podría asociar con una inadecuada condición física, como lo informan los resultados de la Evaluación de Educación Física del Sistema de Medición de Calidad de la Educación (SIMCE) del año 2011; donde 93\% de la población escolar de $8^{\circ}$ básico a nivel nacional presentó esta condición (34), la que llevaría a una inactividad física, ge- 
nerando una pérdida de masa muscular por desuso y afectaría negativamente la funcionalidad muscular (35), ocasionando cuadros de sarcopenia con consecuencias a nivel metabólico y de riesgo cardiovascular (36-38). En nuestro país esta situación fue reportada por Burrows y cols, en una población de adolescentes (39).

En conclusión, los resultados encontrados en el presente estudio indican que esta población escolar presenta una alta prevalencia de sobrepeso, obesidad, RCV y componente graso, más acentuado en los de menor edad y en varones. Relevante era el notable déficit del componente muscular en esta población, que fue más pronunciado en los de mayor edad y en mujeres. Estos antecedentes confirman que esta población está potencialmente en riesgo de contraer a temprana edad enfermedades no transmisibles y sus complicaciones. Es urgente la necesidad de intervenir y vigilar la situación nutricional de los escolares, evaluando su estado nutricional, composición corporal y funcionalidad muscular, con mediciones económicas y aplicables a grupos representativos de la población; obteniendo datos confiables, con personal entrenado y usando una referencia y puntos de corte estandarizados, para comparar y medir la evolución real de esta problemática en esta población.

\section{RESUMEN}

Objetivo: Determinar el estado nutricional y composición corporal en escolares de un colegio de La Serena. Sujetos y Métodos: Estudio transversal en 497 escolares entre 6 y 15 años. Se calculó índice de masa corporal e indicadores de composición corporal. Resultados: La prevalencia de obesidad fue $19,7 \%$, mayor en varones $(24,4 \% ; p=0,010)$, y el sobrepeso $24,3 \%$, mayor en mujeres $(26,7 \% ; p=0,264)$. El exceso del componente graso fue $37,6 \%$, mas prevalente en el $1^{\circ}$ ciclo $(50,0 \% ; p<0,001)$. El déficit de masa muscular fue $31,8 \%$, mayor en mujeres $(36,2 \% ; p=0,048)$ y en el $2^{\circ}$ ciclo $(36,9 \%$; $\mathrm{p}=0,002$ ). En cuanto al riesgo cardiovascular, medido por la circunferencia de cintura, fue de $17,7 \%$, siendo levemente mayor en varones (18,5\%; $p=0,794)$. Conclusión: Existe alta prevalencia en las variables estudiadas en estos escolares. Vigilar la situación nutricional e intervenir es indispensable para prevenir la aparición a temprana edad de enfermedades no transmisibles.

Palabras clave: sobrepeso; obesidad; composición corporal; La Serena; escolares.

Agradecimientos: Los autores desean agradecer al Director del Colegio Adventista de La Serena, Sr. Rolando Montoya Sierra, y al inspector encargado de salud, Sr. Iván Honores, por su disposición y colaboración permanente en la obtención de los datos. Igualmente al Departamento de Salud Pública del la Facultad de Medicina de la Universidad Católica del Norte, por facilitar los equipos y los recursos necesarios para realizar este estudio.

\section{BIBLIOGRAFÍA}

1. World Health Organization. Obesity: preventing and managing the global epidemic. Report of a WHO Consultation. Geneva: World Health Organization; (WHO technical report series 894). 2000.

2. Serdula $M K$, Ivery $D$, Coates $R J$, Freedman DS, Williamson $D F$, Byers T. Do obese children become obese adults? A review of the literature. Prev Med. 1993; 22(2): 167-77.

3. Epidemiology Subcommittee, Committee on Nutrition. Consensus on risk factors for cardiovascular disease in children. Obesity. Arch Argent Pediatr. 2005;103(3):262-78.
4. Must A, Jacques PF, Dallal GE, Bajema CJ, Dietz WH. Longterm morbidity and mortality of overweight adolescents: $A$ follow-up of the Harvard Growth Study of 1922 to 1935. N Engl J Med. 1992; 327(19): 1350-5.

5. Ministry of Health. Nutritional Assessment Technical Standard for Children 6 to 18 years. Available from: www. minsal.cl

6. Chiquete E, Ruiz-Sandoval JL, Ochoa-Guzmán A, SánchezOrozco LV, Lara-Zaragoza EB, Basaldúa N, et al. The Quételet index revisited in children and adults. Endocrinol Nutr. 2014; 61(2):87-92.

7. Treche HM, García AM. Methods for assessing body composition in humans. Biochemical indicators for the assessment of nutritional status. Editorial La Habana Cuba. FACES; 1996: 11-89.

8. Sun SS, Liang $R$, Huang TT, Daniels SR, Arslanian S, Liu $K$, Grave GD, Siervogel RM. Childhood obesity predicts adult metabolic syndrome: the Fels Longitudinal Study. J Pediatr. 2008; 152(2): 191-200.

9. Morrison JA, Friedman LA, Wang P, Glueck CJ. Metabolic syndrome in childhood predicts adult metabolic syndrome and type 2 diabetes mellitus 25 to 30 years later. J Pediatr. 2008; 152(2): 201-6.

10. Freedman DS, Serdula MK, Srinivasan SR, Berenson GS. Relation of circumferences and skinfold thicknesses to lipid and insulin concentrations in children and adolescents: the Bogalusa Heart Study. Am J Clin Nutr. 1999; 69(2):308-17.

11. Moreno MI. Waist circumference: an important and useful measure of cardiometabolic risk. Rev Chil Cardiol. 2010; 29(1): 85-7.

12. Frisancho AR. New norms for upper limb fat and muscle areas for assessment of nutritional status. Am J Cli Nutr. 1981; 34(11):2540-5.

13. Report Nutritional Map of National School Association for Scholarships (JUNAEB). Available from: www.junaeb. cl/wp-content/uploads/2013/03/Informe-Mapa-Nutricional-2013.pdf

14. Bancalari R, Díaz C, Martínez-Aguayo A, Aglony A, Zamorano J, Cerda V, et al. Prevalence of hypertension in school age children and its association with obesity. Rev Med Chil. 2011; 139(7): 872-9.

15. Mardones F, Arnaiz P, Barja S, Giadach C, Villarroel L, Domínguez $A$, et al. Nutritional status, metabolic syndrome and insulin resistance in children from Santiago (Chile). Nutr Hosp. 2013; 28(6):1999-2005.

16. Eyzaguirre CF, Mericq GV, Ceresa OS, Youlton RR, Zacarías SJ. Prevalence of overweight (OW) and obesity $(O)$ in children attending the paedriatric unit of the Clinic Las Condes. Rev Chil Pediatr. 2005; 76(2): 143-9.

17. Kain B, Vio D, Leyton $D$, Cerda $R$, Olivares $C$, Uauy $D$, et al. School- based health promotion intervention for primary schoolchildren from Casablanca, Chile. Rev Chil Nutr. 2005; 32(2):126-32

18. Olivares S, Kain J, Lera L, Pizarro F, Vio F, Morón C. Nutritional status, food consumption and physical activity among Chilean school children: a descriptive study. Eur J Clin Nutr. 2004; 58: 1278-85.

19. Ministry of Education. Construction methodology Socioeconomic Groups 2011. Unit Curriculum and Evaluation Ministry of Education, 2012. Available from: www. agenciaeducacion.cl/wp-content/uploads/2013/02/ Metodologia-de-Construccion-de-Grupos-Socioeconomicos-SIMCE-2011.pdf

20. Cook S, Weitzman M, Auinguer P, Nguyen M, Dietz W. 
Prevalence of a metabolic syndrome Phenotype in adolescents: findings from the third National Health and Nutrition Examination Survey, 1988-1994. Arch Pediatr Adolesc Med. 2003; 157(8): 821-7.

21. Ministry of Health. National Health Survey 2009-2010. Santiago: Ministry of Health, 2011.

22. Ulloa N, Sapunar J, Bustos P, Sáez k, Asenjo S, Taibo M, et al. Frequency of obesity and overweight among school age children living in southern Chile. Rev Med Chil. 2010; 138(11): 1365-72

23. Atalah SE, Loaiza S, Taibo M. Nutritional status of Chilean students according to the NCHS and WHO reference 2007. Nutr Hosp. 2012; 27(1): 1-6

24. Schonhaut L, Rodriguez L, Pizarro T, Kohn J, Merino $D$, López $A$, et al. Concordance in nutritional diagnosis between the healthcare and school teachers teams, using the body mass index (BMI) in the borough of Colina. Rev Chil Pediatr. 2004; 75(1):32 -5.

25. Alarcón AM, Atalah SE. Reliability of anthropometric the measurements in students from Vicuña county (Chile). Rev Chil Nutr. 2009; 36(4): 1056-62.

26. Burrows R, Díaz N, Muzzo S. Variations of body mass index (BMI) according to degree of pubertal development. Rev Med Chil. 2004; 132(11):1363-8.

27. Kain J, Leyton B, Concha F, G Weisstaub, Lobos L, Bustos $N$, et al. Evaluation of an intervention in nutrition education and physical activity to prevent childhood obesity in public schools in Santiago de Chile. Arch Latinoam Nutr. 2012; 62 (1): 60-7.

28. Pucciarelli HM, Cernesen FR, Pinotti LV, Guimarey LM, Giocochea AS. Sexual dismorphism in schoolchildren of the Villa IAPI neighborhood (Quilmes, Buenos Aires, Argentina). Am J Phys Anthropol. 1993; 92(2): 165-72.

29. Jaworski M, Kulanga Z, Pludowski P, Grajda A, Gurzkowska $B$, Napieralska $E$, et al. Population-based centile curves for triceps, subscapular, and abdominal skinfold thicknesses in Polish children and adolescents--the OLAF study. Eur J
Pediatr. 2012; 171(8):1215-21.

30. González JE, Aguilar CM, García LP, Schmidt RJ, García GC. Analysis of the nutritional state and body composition of school children in Granada (Spain). Nutr Hosp. 2012; 27(5): 1496-504

31. Rojas J, Vasquéz L, Sanchéz G, Banik S, Argaéz J. Hands dynamometry in students from Merida, Mexico. Rev Chil Nutr. 2012; 39(3): 45-51.

32. Casajús JA, Leiva MT, Ferrando JA, Moreno L, Aragonés MT, Ara I. Relationship between cardiovascular fitness and fat distribution in children and adolescents. Apunts Med Esport. 2006; 41(149):7-14.

33. Tejedor GV, Nogales EA. Arm fat and muscle area results obtained during a pre-school nutritional evaluation in Madrid. An Esp Pediatr. 1997; 46(4): 335-43.

34. Ministry of Education. National Report of Physical Education Results. Santiago, 2011. Available from: www.agenciaeducacion.cl/wp -content/files_mf/informederesultadosed. física2011.pdf.

35. Jackman RW, Kandarian SC. The molecular basis of skeletal muscle atrophy. Am J Physiol Cell Physiol. 2004; 287(4): C834-43.

36. Rolland Y, Czerwinski S, Abellan Van Kan G, Morley JE, Cesari M, Onder G, et al. Sarcopenia: Its assessment, etiology, pathogenesis, consequences and future perspectives. J Nutr Health Aging. 2008; 12 (7):433-50.

37. Nazmi A, Victora CG. Socioeconomic and racial/ethnic differentials of C-reactive protein levels: a systematic review of population based studies. BMC Public Health. 2007; $7: 212$.

38. Ramírez-Vélez $R$, Da Silva-Grigoletto $M E$, Fernández JM. Current evidence of physical exercise interventions in cardiovascular risk factors. Rev Andal Med Deporte. 2011; 4(4):141-51

39. Burrows $R$, Correa-Burrows $P$, Reyes $M$, Blanco $E$, Albala $C$, Gahagan S. High cardiometabolic risk in healthy Chilean adolescents. Public Health Nutr. 2015: 1-8. 